\title{
¿Nadan en la Abundancia los Jóvenes Españoles y Alemanes? Un Estudio Empírico Sobre la Competencia Fraseológica
}

\author{
Isabel Andúgar Andreu \\ Universitat Jaume I, Castelló de la Plana 12071, España \\ isandugar@gmail.com
}

\begin{abstract}
La presente comunicación se enmarca en el ámbito de la fraseología contrastiva español-alemán. Partiendo de la noción de equivalencia entre unidades fraseológicas (UF), surge la necesidad de disponer de datos reales de competencia de los jóvenes de ambas comunidades lingüísticas. Para ello se realiza un estudio empírico, utilizando un corpus de expresiones fraseológicas equivalentes como Coger el toro por los cuernos $\approx$ den Stier bei den Hörnern packen. El proceso de investigación consta de una revisión previa de diferentes aspectos sobre la disciplina y del concepto de equivalencia, y posteriormente se sientan las bases metodológicas de la investigación empírica. Esta investigación se desarrolla en diferentes fases: el planteamiento de hipótesis, la selección del corpus de UF, la redacción del cuestionario, la selección de informantes, la recogida de datos y análisis de los mismos. Hay que destacar que, en la presente investigación se ha confeccionado un corpus de UF ad hoc, conocido y utilizado por los hablantes nativos de ambas lenguas. Una vez completada la investigación, se procede al análisis cuantitativo y cualitativo de los datos mediante herramientas estadísticas, del que se obtienen datos esclarecedores sobre las diferencias, pero también inesperadas similitudes entre ambas poblaciones. El desarrollo de este tipo de investigaciones es un instrumento muy valioso para establecer posibles mínimos fraseológicos en la didáctica de las lenguas, tanto de las maternas como de las lenguas extranjeras, en la traducción, y por supuesto en la elaboración de herramientas como diccionarios.
\end{abstract}

Keywords: Fraseología contrastiva español-alemán, Unidad fraseológica (UF), Competencia, Corpus, Mínimo fraseológico

\section{Introducción}

\subsection{Planteamiento de la investigación}

La presente comunicación ofrece un análisis de la competencia fraseológica de los jóvenes de las dos comunidades de habla, la española y la alemana, en su lengua materna. 
Es generalmente asumido que la competencia lingüística es la disponibilidad activa o pasiva de determinadas estructuras que poseen los hablantes de una lengua: es decir, no solo qué unidades lingüísticas son capaces de usar (competencia activa), sino también aquellas que conocen, aunque no suelan utilizar (competencia pasiva). ${ }^{1}$ En el caso que nos ocupa, el objetivo es realizar un estudio empírico que nos arroje datos especialmente cuantitativos, pero no sólo, acerca del conocimiento de expresiones fraseológicas que poseen los jóvenes.

Las expresiones fraseológicas en cualquiera de sus formas (colocaciones, locuciones, refranes, etc.) son un componente importante de las lenguas, no sólo por su frecuencia de aparición, sino también por su complejidad, pues a diferencia de lo que ocurre con las palabras, la consulta de las unidades fraseológicas (UF) en los diccionarios no resulta tarea fácil. En definitiva, si la correcta utilización de la fraseología no es sencilla para los hablantes nativos de una lengua, es mucho más difícil para quienes intentan dominar el sistema fraseológico de una segunda lengua, pues no sólo se trata de esquemas lingüísticos diferentes, sino también de referentes culturales distintos.

Si bien es frecuente encontrar estudios lingüísticos que investigan la competencia lingüística en cuanto a la disponibilidad léxica en general, tanto en español como en alemán, es decir, el caudal de vocabulario que activa o pasivamente conoce un grupo de hablantes, no podemos decir lo mismo de la competencia o disponibilidad fraseológica en general, que cuenta con un número de trabajos especializados sensiblemente menor. ${ }^{2}$

Tal vez esto se deba a que la misma investigación fraseológica es relativamente joven comparada con la lingüística en general, pues debemos remontarnos apenas a las últimas décadas para presenciar un desarrollo importante de los estudios fraseológicos, no solo desde una perspectiva teórica, sino también aplicada, de manera que actualmente podemos considerar la situación de la disciplina bastante consolidada.

Aun así, la competencia fraseológica ha sido poco estudiada empíricamente, si exceptuamos los refranes. También es escasa la investigación de la competencia que relaciona los sistemas fraseológicos de lenguas diferentes. ${ }^{3}$ Precisamente esta ausencia de trabajos al respecto ha motivado la presente investigación, en la que se intenta dilucidar qué competencia tienen los jóvenes en su lengua materna a partir de un corpus fraseológico equivalente. Nos interesa especialmente averiguar si: a) la competencia es similar en ambas lenguas contrastadas, y b) algunos factores sociológicos pueden condicionar esa competencia.

1 Yaguello (1983: 79-80) se refiere a la competencia pasiva como la «aptitud para reconocer», y la competencia activa a la «capacidad de reproducir».

2 No obstante, se han realizado algunos estudios en lo que respecta a refranes, por ejemplo, las investigaciones realizadas por Sevilla para el español (Sevilla, 2010).

3 Los estudios contrastivos de fraseología abordan generalmente aspectos sobre la lengua literaria, el análisis de corpus y sus traducciones (López Roig, 2002), o las equivalencias entre las lenguas (Tarnovska, 2004). 


\subsection{Metodología}

La investigación aúna las vertientes lingüística y sociolingüística, así pues su base metodológica está determinada por preceptos de las dos disciplinas, como no podía ser de otra manera. Las fases de la investigación se desarrollan atendiendo a la secuencia siguiente: la elaboración del corpus de UF equivalente, la redacción del cuestionario, la selección de informantes y la recogida de datos.

Elaboración del corpus equivalente. Además de cumplir con el criterio previo de equivalencia en la comparación interlingüística español-alemán, se considera que las UF integradas en el corpus deben cumplir otros requisitos, como representatividad, pues deben ser UF conocidas ampliamente por los hablantes nativos jóvenes en cada una de las muestras, y adecuación, que hace referencia puesto que la forma externa de la UF no venía dada con antelación en un texto fijado, era necesario una verificación de la misma para garantizar el rigor de la investigación. La representatividad de las UF se decidió a partir de estudios previos, en los que se verificó un grado de conocimiento superior al $50 \%{ }^{4}$ Además, la adecuación de la forma elegida se realiza a partir del cotejo de diferentes fuentes fraseográficas, comprobando que la variante elegida por nosotros fuera la forma más usual o 'canónica', según denominación de Conca y Guia (2014).

El corpus de UF es el siguiente:

UF1 poner los cuernos $\approx$ Hörner aufsetzen

UF2 poner la mano en el fuego $\approx$ die Hand ins Feuer legen

UF3 como el perro y el gato $\approx$ wie Hund und Katze

UF4 no tener pelos en la lengua $\approx$ kein Blatt vor den Mund haben

UF5 ponerse los pelos de punta $\approx$ jemandem stehen die Haare zu Berge

UF6 descubrir la pólvora $\approx$ das Pulver erfunden haben

UF7 tirar la casa por la ventana $\approx$ das Geld zum Fenster hinauswerfen

UF8 sonar a chino $\approx$ Spanisch vorkommen

UF9 ser todo oídos $\approx$ ganz Ohr sein

UF10 hacerse la boca agua das Wasser im Mund zusammenlaufen

UF11 llamar a las cosas por su nombre $\approx$ die Dinge bei ihrem Namen nennen

UF12 echar leña al fuego $\approx$ Öl ins Feuer giessen

UF13 pagar con la misma moneda $\approx$ mit gleicher Münze heimzahlen

UF14 no tener pies ni cabeza weder Hand noch Fuss haben

UF15 matar dos pájaros de un tiro z zwei Fliegen mit einer Klappe schlagen

UF16 faltar un tornillo $\approx$ nicht alle Tassen im Schrank haben

UF17 amor a primera vista $\approx$ Liebe auf den ersten Blick

4 Tarnovska (2004) ha considerado también este porcentaje como un buen nivel de competencia paremiológica. Al igual que ocurre en nuestro trabajo, Tarnovska desarrolló su investigación empírica por medio de encuestas, a fin de delimitar un refranero básico español (unas 250 paremias). Este trabajo lo completa con el análisis contrastivo de otras unidades fraseológicas equivalentes en ruso y ucraniano. La finalidad de este trabajo consiste en acercar las paremias al estudiante de español como lengua extranjera, estableciendo un mínimo paremiológico español. 
UF18 tener algo en la punta de la lengua etwas auf der Zunge liegen

UF19 nadar en la abundancia $\approx$ im Geld schwimmen

UF20 perder hasta la camisa $\approx$ bis aufs Hemd ausziehen

UF21 meter la cuchara $\approx$ seinen Senf dazu geben

UF22 estar en el séptimo cielo $\approx$ im siebten Himmel sein

UF23 ser la oveja negra de la familia $\approx$ das schwarze Schaf der Familie sein

UF24 ser un lobo con la piel de cordero $\approx$ ein Wolf im Schafspelz sein

UF25 tener sangre azul blaues Blut haben

UF26 no ser carne ni pescado $\approx$ weder Fisch noch Fleisch

UF27 nadar a contracorriente $\approx$ gegen den Strom schwimmen

UF28 saber dónde le aprieta el zapato a alguien $\approx$ wissen, wo einen der Schuh

drückt

UF29 tender un puente de plata $\approx$ eine goldene Brücke bauen

UF30 perder el hilo $\approx$ die Faden verlieren

UF31 jugar con las cartas boca arriba $\approx$ mit offenen Karten spielen

UF32 llevar los pantalones $\approx$ die Hosen anhaben

UF33 hacer la corte a alguien $\approx$ jemandem den Hof machen

UF34 llevar una venda en los ojos $\approx$ ein Brett vor dem Kopf haben

UF35 ser el cuento de la lechera eine Milchmädchenrechnung sein

UF36 estar en boca de todos $\approx$ in aller Munde sein

UF37 estar hasta las narices $\approx$ die Nase voll haben

UF38 construir sobre arena $\approx$ auf Sand bauen

UF39 poner la zancadilla a alguien $\approx$ jemandem ein Bein stellen

UF40 ponerle los nervios de punta a alguien $\approx$ jemandem auf die Nerven gehen

UF41 hacer una montaña de un grano de arena $\approx$ aus einer Mücke einen Elefanten machen

UF42 tener gato encerrado $\approx$ die Katze im Sack kaufen

UF43 levantarse con el pie izquierdo $\approx$ mit dem linken Fuß zuerst aufstehen

UF44 sacar las castañas del fuego a alguien $\approx$ jemandem die Kastanien aus dem

Feuer holen

UF45 coger el toro por los cuernos $\approx$ den Stier bei den Hörnern packen

UF46 ser pobre como una rata $\approx$ arm wie ein Kirchenmaus sein

UF47 echar tierra en los ojos a alguien $\approx$ jemandem Sand in die Augen streuen

UF48 encontrar un pelo en la sopa $\approx$ ein Haar in der Suppe finden

UF49 meter las narices en todo $\approx$ die Nase in alles stecken

UF50 enseñarle los dientes a alguien $\approx$ jemandem die Zähnen zeigen

Redacción del cuestionario. Los datos de la investigación son recogidos en un cuestionario elaborado ad hoc, con dos partes diferenciadas, una lingüística, en la que se presentan cincuenta expresiones equivalentes en ambas lenguas y se investiga la competencia que poseen los hablantes, y otra sociológica, en la que se obtienen datos de los sujetos por medio de un breve cuestionario sociológico.

Selección de informantes. La selección de los informantes no se hace de forma manipulada respecto a ninguno de los factores sociales, aunque sí es necesario 
establecer premisas que determinen un punto de partida básico, como es la edad. Para este fin, se han elegido niveles educativos similares en ambas comunidades lingüísticas de manera que al menos estuviera asegurado el rango similar de edades. En ambos casos son niveles de la enseñanza pre-universitaria, entre los 14 y los 17 años. Las muestras se tomaron en Castellón y Hamburgo.

La distribución de los informantes por variables edad y sexo se muestran en las tablas siguientes.

Tabla 1. Edad de los informantes

\begin{tabular}{lccccc}
\hline EDAD (años) & 14 & 15 & 16 & 17 & TOTAL INF \\
ESPAÑOL & 14 & 15 & 15 & 1 & 45 \\
ALEMÁN & 16 & 16 & 5 & 8 & 45 \\
\hline
\end{tabular}

Tabla 2. Sexo de los informantes

\begin{tabular}{lccc}
\hline SEXO & MUJERES & HOMBRES & TOTAL INF \\
ESPAÑOL & 21 & 24 & 45 \\
ALEMÁN & 25 & 20 & 45 \\
\hline
\end{tabular}

Recogida de datos. En el proceso de recogida de datos participaron profesores de lenguas y cada una de las partes del cuestionario tuvo un tiempo limitado.

Posteriormente, se procede al análisis con la ayuda de herramientas estadísticas que facilitan la interpretación de los resultados obtenidos, tanto cuantitativa como cualitativamente. Entre otras posibilidades, el programa SPSS (Statistical Package for Social Sciences), versión 22, se erige como una buena elección, pues se trata de un programa estadístico muy utilizado en investigaciones relacionadas con las ciencias sociales, como la psicología o la medicina, en las que se analizan las estadísticas en relación a datos sociológicos como el sexo, la edad, etc. Este tipo de datos también aparecen en este estudio, por ello la conveniencia de su utilización.

Las variables que se manejan en la investigación corresponden a variables independientes y variables dependientes. Las variables independientes son aquellas que vienen dadas en los sujetos $\mathrm{y}$, a su vez, pueden condicionar la variable dependiente.

Las variables independientes utilizadas en la investigación son idioma (español, alemán), edad $(14,15,16,17)$ y sexo (mujer, hombre). La variable dependiente, aquella sobre la cual queremos ver la incidencia de las otras, es la competencia fraseológica.

Las operaciones principales aplicadas para el análisis de los datos fueron de varios tipos. Por un lado, se utilizaron medidas de tendencia central, es decir, medidas que agrupan los datos obtenidos, y por lo tanto son valores que resultan representativos de todos los valores que toma la variable. Entre estas podemos destacar la media, que supone la media aritmética de todos los valores. 
Para poder observar además si los valores que ofrecen los análisis son significativos se utilizan también diferentes métodos, de los cuales hemos empleado, entre otros, los estadísticos conocidos como T-Student. Puesto que en el entorno lingüístico lo primordial es facilitar una interpretación visual, se ofrecen a continuación los datos.

\section{Datos Relevantes}

En la investigación se plantea una hipótesis previa: hay diferencias en la competencia fraseológica global de los jóvenes. A partir del análisis de los datos se comprobará si se valida o refuta. Pasamos a exponerlos a continuación.

\subsection{La Competencia Fraseológica Global de los Jóvenes}

Las medias de competencia obtenidas en ambas muestras se observan a continuación (Tabla 3):

Tabla 3. Nivel de competencia global de las muestras española y alemana

\begin{tabular}{|c|c|c|c|c|c|}
\hline \multicolumn{6}{|c|}{ Estadísticos de grupo } \\
\hline & IDIOMA & $\mathrm{N}$ & Media & $\begin{array}{l}\text { Desviación } \\
\text { típ. }\end{array}$ & $\begin{array}{l}\text { Error } \\
\text { típ. de la } \\
\text { media }\end{array}$ \\
\hline COMPETENCIA & ESPAÑOL & 45 & 30,11 & 7,493 & 1,117 \\
\hline $\begin{array}{l}\text { FRASEOLÓGICA } \\
\text { GLOBAL }\end{array}$ & ALEMÁN & 45 & 28,02 & 6,538 & ,975 \\
\hline
\end{tabular}

De las cincuenta UF analizadas en el cuestionario, los estudiantes españoles muestran una media de competencia de 30,11 UF, mientras que los alemanes tienen una competencia del 28,02 UF. Aunque se observa diferencia de competencia, a priori se desconoce si esta puede ser significativa. Para comprobarlo, se realiza una prueba Tde Student (Tabla 4). Se trata de una prueba de contraste estadístico que considera las diferencias de medias entre dos muestras que son independientes entre sí como en este caso.

Tabla 4. Nivel de competencia global: Prueba T (1)

\begin{tabular}{|c|c|c|c|c|}
\hline \multicolumn{5}{|c|}{ Prueba de muestras independientes } \\
\hline & & $\begin{array}{l}\text { Prueba de Levene para la } \\
\text { igualdad de varianzas }\end{array}$ & \multicolumn{2}{|c|}{$\begin{array}{l}\text { Prueba T para la } \\
\text { igualdad de medias }\end{array}$} \\
\hline & & $\mathrm{F}$ & Sig. & $\mathrm{T}$ \\
\hline \multirow{2}{*}{$\begin{array}{l}\text { TOTAL } \\
\text { COMPETENCIA }\end{array}$} & Se han asumido varianzas iguales & 377 & ,541 & $-1,409$ \\
\hline & No se han asumido varianzas iguc & & & $-1,409$ \\
\hline
\end{tabular}


FRASEOLÓGICA

Y a continuación se observa si es significativa (Tabla 5):

Tabla 5. Nivel de competencia global: Prueba T (2)

\begin{tabular}{lcccc}
\hline \multicolumn{4}{c}{ Prueba de muestras independientes } \\
& Prueba T para la igualdad de medias & Gl & $\begin{array}{c}\text { Sig. } \\
\text { (bilat- }\end{array}$ & $\begin{array}{c}\text { Diferencia } \\
\text { de medias }\end{array}$ \\
& & & eral) & \\
TOTAL & Se han asumido varianzas iguales & 88 &, 162 & $-2,089$ \\
COMPETENCIA & No se han asumido varianzas iguales & 86,414 &, 162 & $-2,089$ \\
FRASEOLÓGICA & & & &
\end{tabular}

Los análisis indican que, al menos con la muestra disponible en nuestro estudio, no hay diferencias entre los resultados de ambas poblaciones: $\mathrm{T}=-1,40$ (Tabla 4), ya que ofrece una valor sig.=,162 (Tabla 5), mayor de ,05. Esto significa que, aunque las medias son distintas, esa diferencia de medias no resulta significativa.

Puesto que ambas comunidades son distintas, tanto lingüística como culturalmente, cabía esperar diferencias significativas entre la competencia mostrada por los alumnos españoles y alemanes. Sin embargo, los jóvenes españoles y alemanes presentan una competencia muy similar en su lengua nativa.

\section{Conclusiones}

Del mismo modo que un estudio sobre disponibilidad léxica ofrece datos interesantes sobre el uso del idioma por parte de la población que lo sustenta, entendemos que este tipo de estudio en el campo fraseológico puede ser también de gran ayuda, pues no solo arrojará luz sobre el uso real de fraseología en las comunidades lingüísticas respectivas y se comprobará si las expresiones más usadas en una comunidad coinciden en la otra, sino que, además, verificará si esa competencia es socialmente homogénea o por el contrario depende de ciertos factores sociales.

El objetivo perseguido con este tipo de investigaciones no acaba con la descripción de la situación, sino que la información lingüística obtenida puede ser utilizada en posteriores estudios y permite a su vez establecer mínimos fraseológicos, tan útiles para trabajar en situaciones de interacción lingüística como son la docencia de lenguas extranjeras o la traducción, y también para elaborar las herramientas necesarias de estas dos disciplinas lingüísticas: diccionarios fraseológicos que atiendan a criterios de uso efectivo, indispensables cuando se trata de contrastar estas expresiones.

Desde esta pequeña contribución esperamos sentar las bases para realizar estudios más amplios a fin de disponer de datos más representativos sobre muestras de población mayores. 


\section{Referencias}

1. Yaguello, M.: Alicia en el país del lenguaje, Mascarón, Madrid (1983).

2. Sevilla Muñoz, J.: «La competencia paremiológica en la generación española de más de 65 años», Phraseologie global - areal - regional, Jarmo Korhonen, Wolfgang Mieder, Elisabeth Piirainen, Rosa Piñel (eds.). Helsinki: Universität Helsinki, pp. 151-158 (2010).

3. López Roig, C.: Aspectos de Fraseología (alemán-español) en el sistema y en el texto, Peter Lang, Frankfurt a. M. (2002).

4. Tarnovska, O.: Refranero básico español con correspondencias en ruso y ucraniano, Logos, Kiev (2004).

5. Conca, M. y J. Guia: La fraseologia. Principis, mètodes $i$ aplicacions, Bromera, Valencia (2014). 